\title{
DERIVING CRITERION WEIGHTS FROM EXPERT JUDGEMENTS: SIMPLE AND FUZZY RANKINGS VS SAATY'S PREFERNCE SCALE
}

\author{
Sergey Kadenko* \\ National Academy of Sciences of Ukraine \\ The Institute for Information Recording \\ Kyiv, Ukraine \\ E-mail: sergeykadenko@mail.ru
}

\begin{abstract}
An approach to alternative estimation criterion weights' calculation based on multi-criteria alternative rankings is suggested. It is assumed that in case it is problematic for experts to estimate alternatives and criterion weights cardinally and/or build respective pair comparison matrices while decisionmaking, it's possible to find criterion weights based on previous experience of ordinal alternative estimation according to given criteria. Criterion weight calculation method and ways of its extension to fuzzy rankings, as well as several method modifications are considered.
\end{abstract}

Keywords: criterion, ordinal estimate, ranking, fuzzy ranking, weight coefficient, pair comparison.

\section{Introduction}

A special place in expert estimation and decision-making support belongs to ordinal estimates, or rankings. In order to avoid further confusion let us first define an ordinal estimate of an alternative or an object as its number in a set of alternatives. In contrast to cardinal estimates, ordinal estimates (or rankings) do not bear any specific information about quantitative relation between alternatives, allowing only to order alternatives according to a given criterion. If it is problematic for the experts to estimate criterion weights directly, it is worth trying to calculate these weights based on previous experience of alternative estimation according to these same criteria. Besides, results of such calculations may be more relevant and objective than the results of direct weight estimation, since they reflect the whole experience of experts' "acquaintance" with the given domain and not only their current judgements. The problem of criterion weights definition based on multi-criteria alternative rankings was posed and addressed in (Kadenko 2009, 2010). The key issues to be addressed in this paper are: 1) formulation and solution of a similar problem in case expert alternative estimates are given in the form of fuzzy rankings 2) comparing the results of weight calculation methods using fuzzy and non-fuzzy alternative rankings as initial data and 3) suggesting ways of simplifying the alternative estimation process and making the expertise results more adequate.

\section{Problem formulation}

What is given (initial data): 1) A set of alternatives (objects) $A_{1}, A_{2}, \ldots, A_{m} ; 2$ ) A set of preferentially independent criteria of alternative estimation, which are compatible with each other $C_{1}, C_{2}, \ldots, C_{n} ; 3$ ) Ranking of alternatives according to each criterion $\left\{r_{i j}\right\}, i=1 . . m, j=1 . . n$, where $r_{i j}$ is a rank score of the $i$-th alternative according to the $j$-th criterion; 4) Strict global ranking (total order) of alternatives (alternatives' ordinal estimates according to a global criterion $G) g_{1}, \ldots, g_{m}$

We should find: a set of normalized alternative estimation criterion weights: $\left\{w_{j}\right\}, j=1 . . n$; $\sum_{j=1}^{n} w_{j}=1, w_{j}>0, j=1 . . n$, preserving the initial global ranking of alternatives after weighted summing of single-criterion ordinal alternative estimates.

\footnotetext{
* Corresponding author
} 


\section{Solution method overview}

Presumably, the global alternative ranking is a ranking of weighted single-criterion rank sums (aggregation is conducted using weighted Borda method (Totsenko, 2005)), so our task is to find a solution for a system of inequalities of the following type:

$$
\sum_{j=1}^{n} a_{i j} \times w_{j}>0, i=1 . . m-1 ; w_{j}>0, j=1 . . n
$$

where $a_{i j}=r_{i+1, j}-r_{i j}$, presumably, alternatives are rearranged in order of their global ranks' decrease.

$(i=1 . . m(m-1) / 2$ if we suppose that global ranking transitivity requirement may be unfulfilled).

Each inequality corresponds to a pair comparison of two alternatives $A_{i}$ and $A_{j}$. Say, alternative $A_{i}$ dominates $A_{k}$ according to the global criterion $G$. Then $A_{i} \succ A_{k} \Rightarrow g_{i}<g_{k} \Rightarrow \sum_{j=1}^{n} w_{j} r_{i j}<\sum_{j=1}^{n} w_{j} r_{k j} \Rightarrow \sum_{j=1}^{n} w_{j}\left(r_{k j}-r_{i j}\right)>0 \Rightarrow \sum_{j=1}^{n} a_{l j} \times w_{j}>0$, where $a_{l j}=r_{k j}-r_{i j}$ and $l$ is a respective inequality number in system (1). In general case the problem can be solved using the weight acceptability area search method described in (Kadenko 2009, 2010).

In case we assume that mono-criterion rankings are aggregated according to modified Condorcet (and not Borda) method (Totsenko, 2005), solution procedure will be slightly different from the one outlined above. The difference lies in the way the inequalities are formed. As we know, in Condorcet's method domination matrices corresponding to all the rankings are built. Each element $d_{i j}$ of a domination matrix $D=\left\{d_{i j}, i, j=1 . . m\right\}$ corresponding to a ranking $R=\left\{r_{i}, i=1 . . m\right\}$ represents an ordinal pair comparison of alternatives $A \mathrm{i}$ and $A_{j}$. It is defined as follows:

$$
d_{i j}=1, A_{i} \succ A_{j} ; d_{i j}=-1, A_{i} \prec A_{j} ; d_{i j}=0, A_{i} \equiv A_{j}
$$

In order to get an aggregate preference relation according to "weighted" Condorcet method, it is necessary to conduct weighted summing of respective mono-criterion ordinal pair comparisons $d_{i j}$ (and not the ranks of respective alternatives as in "weighted" Borda method). So the inequalities system will be formed according to the following rule. Suppose, $A_{i}$ dominates $A_{k}$ according to the global criterion $G$.

Then $A_{i} \succ A_{k} \Rightarrow g_{i}<g_{k} \Rightarrow \sum_{s=1}^{m} d_{i s}^{(G)}>\sum_{s=1}^{m} d_{k s}^{(G)} \Rightarrow \sum_{j=1}^{n} w_{j} \sum_{s=1}^{m}\left(d_{i s}^{(j)}-d_{k s}^{(j)}\right)>0 \Rightarrow \sum_{j=1}^{n} a_{l j} \times w_{j}>0$,

where $a_{l j}=\sum_{s=1}^{m}\left(d_{i s}^{(j)}-d_{k s}^{(j)}\right)$ and $l$ is a respective inequality number in system (1).

\section{Fuzzy rankings' case}

\subsection{Fuzzy ranking procedure}

In case when an expert, building alternative rankings, is not sure about ordinal relation between alternatives, preference relations can be represented by fuzzy rankings. In this paper we shall mostly refer to fuzzy number ranking procedures suggested by (Tseng \& Klein, 1989). Fuzzy preference relation is defined as a relation between two fuzzy numbers $A$ and $B$, which can either overlap (i.e. their membership function definition areas overlap) or not overlap with each other. In overlap areas fuzzy numbers are considered indifferent. In non-overlap areas either $A$ or $B$ is considered dominant. Fuzzy preference relations between two numbers $A$ and $B$ given by (Tseng \& Klein, 1989) and later cited and used by (Jiao \& Tseng, 1998) for product design concept evaluation are calculated according to the following formulas:

$$
R(A, B)=\frac{S(A, B)+S(A \cap B, 0)}{S(A, 0)+S(B, 0)} ; R(B, A)=\frac{S(B, A)+S(B \cap A, 0)}{S(A, 0)+S(B, 0)}
$$




\section{S. Kadenko, Criterion Weights}

where $S(A, B)$ denotes the areas where $A$ dominates $B, S(B, A)$ denotes the areas where $B$ dominates $A$, $S(A, 0)$ denotes the area of $A, S(B, 0)$ denotes the area of $B, S(A \cap B, 0)$ denotes the overlap area where $A$ and $B$ are indifferent. To build a mono-criterion fuzzy ordering relation on a set of $m$ alternatives (Jiao $\&$ Tseng, 1998) suggest using a matrix form: $\left[P\left(A_{i}, A_{j}\right)\right\rfloor_{m \times m}$, where each matrix element represents the value of fuzzy preference relation between alternatives $A_{i}$ and $A_{k}$, which, in turn, lies within the limits of the interval $[0,1]$. The mono-criterion alternative estimate aggregation formula used by (Jiao \& Tseng, 1998) looks as follows:

$$
p^{\prime \prime}\left(A_{i}, A_{k}\right)=\left\{\begin{array}{l}
\frac{\sum_{j=1}^{n} w_{j}\left\{\max \left(\left(P\left(A_{i}, A_{k}\right)-0.5\right), 0\right)\right\}}{\sum_{j=1}^{n} w_{j}\left|P\left(A_{i}, A_{k}\right)-0.5\right|}, i \neq k \\
0.5, i=k
\end{array}, i \neq k\right.
$$

where $n$ is the number of alternative estimation criteria and $w_{j}$ is the relative weight of $j$-th criterion.

The formulation of such aggregation rule for fuzzy ordinal estimates enables us to try to pose the problem, inverse to mono-criterion ranking aggregation one. The suggested problem statement is given below.

\subsection{Problem statement and solution idea: fuzzy ranking case} What is given (initial data):

1) A set of alternatives (objects) $\left.A_{1}, A_{2}, \ldots, A_{m} ; 2\right)$ A set of preferentially independent criteria of alternative estimation, which are compatible with each other $\left.C_{1}, C_{2}, \ldots, C_{n} ; 3\right)$ Mono-criterion "fuzzy" ranking matrices $\left\{d_{i k}^{j}\right\}, i, k=1 . . m, j=1 . . n$, where $d_{i k}^{j}$ is a value of preference relation between $i$-th and $k$-th alternatives according the $j$-th criterion. $d_{i k}^{j} \in[0,1] ; d_{i k}^{j}=1-d_{k i}^{j}$; 4) Global fuzzy ranking matrix of alternatives $\left\{g_{i k}\right\}, i, k=1 . . m$ (alternatives" "fuzzy ordering" according to a global criterion $G)$.

We should find: a set of normalized alternative estimation criterion weights $\left\{w_{j}\right\}, j=1 . . n$; $\sum_{j=1}^{n} w_{j}=1, w_{j}>0, j=1 . . n$, preserving the initial global fuzzy ranking of alternatives after monocriterion fuzzy preferences aggregation according to formula (4).

It is not difficult to notice that when $i \neq k$, the aggregation formula (4) can be brought to linear form. To solve the weight calculation problem, formulated above, we need to solve a system of $(m(m-1) / 2)+1$ linear equations with $n$ unknown variables:

$$
\left\{\sum_{j=1}^{n} w_{j} b_{i k}^{j}=0, i, k=1 . . m, i \neq k ; \sum_{j=1}^{n} w_{j}=1 ; w_{j}>0, j=1 . . n\right.
$$

In general case the system will be redundant and have no exact solutions. To solve such a system various methods can be used, such as least squares method, group method of data handling and others.

\section{Numerical example: comparing criterion weights calculated based on fuzzy and non-fuzzy ("defuzzified") rankings}

In order to test the approach described in section 3 of this paper on some real data and, first of all, to compare it with the results of the method based on fuzzy rankings, an example provided by (Jiao \& Tseng, 1998) was used. Their article describes a multi-criteria decision-making problem solution based on fuzzy rankings. The approach suggested by (Jiao \& Tseng, 1998) is illustrated by a specific example, particularly, a heat sink design selection problem. First fuzzy rankings of five heat sink designs are built based on their evaluation according to 4 tangible and 2 intangible criteria. After that criterion weights are defined using the AHP method (Saaty, 2008). Then fuzzy ordering matrices are aggregated and the general rating and ranking of alternatives (i.e. heat sink design variants) are built. Fuzzy and non-fuzzy rankings, as well as ratings of alternatives are provided in the tables 1 and 2. 
Table 1. Fuzzy and non-fuzzy mono-criterion rankings of alternatives (from (Jiao \& Tseng, 1998))

\begin{tabular}{|c|c|c|c|c|c|c|c|c|c|c|c|c|c|c|c|}
\hline \multicolumn{8}{|c|}{ Criterion $C_{1}$ : Thermal resistance } & \multicolumn{8}{|c|}{ Criterion $C_{2}$ : Size-length } \\
\hline & $A_{1}$ & $A_{2}$ & $A_{3}$ & $A_{4}$ & $A_{5}$ & Rating & Rank & & $A_{1}$ & $A_{2}$ & $A_{3}$ & $A_{4}$ & $A_{5}$ & Rating & Rank \\
\hline$A_{1}$ & 0.5 & 0.425 & 0.488 & 0.36 & 0.35 & 1.623 & 5 & $A_{1}$ & 0.5 & 0.114 & 0.367 & 0.197 & 0.236 & 0.914 & 5 \\
\hline$A_{2}$ & 0.575 & 0.5 & 0.498 & 0.318 & 0.39 & 1.781 & 4 & $A_{2}$ & 0.886 & 0.5 & 0.785 & 0.625 & 0.67 & 2.966 & 4 \\
\hline$A_{3}$ & 0.512 & 0.502 & 0.5 & 0.485 & 0.361 & 1.860 & 3 & $A_{3}$ & 0.633 & 0.215 & 0.5 & 0.313 & 0.357 & 1.518 & 1 \\
\hline$A_{4}$ & 0.64 & 0.682 & 0.515 & 0.5 & 0.572 & 2.409 & 1 & $A_{4}$ & 0.803 & 0.375 & 0.687 & 0.5 & 0.552 & 2.417 & 2 \\
\hline$A_{5}$ & 0.65 & 0.61 & 0.639 & 0.428 & 0.5 & 2.327 & 2 & $A_{5}$ & 0.764 & 0.33 & 0.643 & 0.448 & 0.5 & 2.185 & 3 \\
\hline \multicolumn{8}{|c|}{ Criterion $C_{3}$ : Size-width } & \multicolumn{8}{|c|}{ Criterion $C_{4}$ : Cost } \\
\hline & $A_{1}$ & $A_{2}$ & $A_{3}$ & $A_{4}$ & $A_{5}$ & Rating & Rank & & $A_{1}$ & $A_{2}$ & $A_{3}$ & $A_{4}$ & $A_{5}$ & Rating & Rank \\
\hline$A_{1}$ & 0.5 & 0.615 & 0.596 & 0.56 & 0.236 & 2.007 & 2 & $A_{1}$ & 0.5 & 0.5 & 0.5 & 0.5 & 1 & 2.5 & 1 \\
\hline$A_{2}$ & 0.385 & 0.5 & 0.479 & 0.442 & 0.159 & 1.465 & 5 & $A_{2}$ & 0.5 & 0.5 & 0.5 & 1 & 1 & 2 & 2 \\
\hline$A_{3}$ & 0.404 & 0.521 & 0.5 & 0.462 & 0.171 & 1.558 & 4 & $A_{3}$ & 0.5 & 0.5 & 0.5 & 1 & 1 & 2 & 2 \\
\hline$A_{4}$ & 0.44 & 0.558 & 0.538 & 0.5 & 0.182 & 1.718 & 3 & $A_{4}$ & 0.5 & 0 & 0 & 0.5 & 0 & 0.5 & 5 \\
\hline$A_{5}$ & 0.764 & 0.841 & 0.629 & 0.918 & 0.5 & 3.252 & 1 & $A_{5}$ & 0 & 0 & 0 & 1 & 0.5 & 1 & 4 \\
\hline \multicolumn{8}{|c|}{ Criterion $C_{5}$ : Mounting } & \multicolumn{8}{|c|}{ Criterion $C_{6}$ : Compatibility } \\
\hline & $A_{1}$ & $A_{2}$ & $A_{3}$ & $A_{4}$ & $A_{5}$ & Rating & Rank & & $A_{1}$ & $A_{2}$ & $A_{3}$ & $A_{4}$ & $A_{5}$ & Rating & Rank \\
\hline$A_{1}$ & 0.5 & 0 & 0.111 & 0 & 0 & 0.111 & 5 & $A_{1}$ & 0.5 & 1 & 0.5 & 1 & 1 & 2.5 & 1 \\
\hline$A_{2}$ & 1 & 0.5 & 1 & 0.875 & 0 & 2.875 & 2 & $A_{2}$ & 0 & 0.5 & 0 & 0.5 & 0.5 & 1 & 2 \\
\hline$A_{3}$ & 0.889 & 0 & 0.5 & 0.125 & 0 & 1.114 & 4 & $A_{3}$ & 0.5 & 1 & 0.5 & 1 & 1 & 2.5 & 1 \\
\hline$A_{4}$ & 1 & 0.125 & 0.875 & 0.5 & 0 & 2 & 3 & $A_{4}$ & 0 & 0.5 & 0 & 0.5 & 0.5 & 1 & 2 \\
\hline$A_{5}$ & 1 & 1 & 1 & 1 & 0.5 & 4 & 1 & $A_{5}$ & 0 & 0.5 & 0 & 0.5 & 0.5 & 1 & 2 \\
\hline
\end{tabular}

Table 2. Fuzzy and non-fuzzy global ranking of alternatives (from (Jiao \& Tseng, 1998))

\begin{tabular}{|l|l|l|l|l|l|l|l|}
\hline \multicolumn{2}{|c|}{ Criterion G: Global (aggregated) estimate } \\
\hline & $A_{1}$ & $A_{2}$ & $A_{3}$ & $A_{4}$ & $A_{5}$ & Rating & Ranking \\
\hline$A_{1}$ & 0.5 & 0.138 & 0.101 & 0.097 & 0.229 & 0.565 & 5 \\
\hline$A_{2}$ & 0.862 & 0.5 & 0.86 & 0.532 & 0.273 & 2.527 & 2 \\
\hline$A_{3}$ & 0.899 & 0.14 & 0.5 & 0.464 & 0.239 & 1.743 & 4 \\
\hline$A_{4}$ & 0.903 & 0.468 & 0.536 & 0.5 & 0.22 & 2.177 & 3 \\
\hline$A_{5}$ & 0.771 & 0.727 & 0.761 & 0.78 & 0.5 & 3.038 & 1 \\
\hline
\end{tabular}

It is obvious, that if we try to calculate criterion weights based on fuzzy alternative ranking matrices, using the approach provided in section 4 of this paper, we shall obtain exact values, given in Table 3 . The question we are trying to answer is: will the weights, calculated based on non-fuzzy rankings (these are provided in Table 1, in last columns of "sub-tables" with mono-criterion alternative estimates) coincide with, or, at least, be close to those exact values (and in case they will not, why). Weight ranges and average weights (arithmetic mean of weight acceptability range extreme points), calculated based on non-fuzzy rankings, using the approach described in section 3 of this paper, are provided in table 3.

Table 3 Criterion weights calculated based on non-fuzzy rankings

\begin{tabular}{|l|l|l|l|l|l|l|}
\hline Criteria & $C_{1}$ & $C_{2}$ & $C_{3}$ & $C_{4}$ & $C_{5}$ & $C_{6}$ \\
\hline $\begin{array}{l}\text { Weight ranges under presumption that } \\
\text { estimate aggregation is conducted using } \\
\text { Borda method }\end{array}$ & $(0 ; 0.442)$ & $(0 ; 0.667)$ & $(0 ; 0.333)$ & $(0 ; 0.385)$ & $(0 ; 0.745)$ & $(0 ; 0.625)$ \\
\hline $\begin{array}{l}\text { Average weights (weight acceptability area } \\
\text { centre calculated as arithmetic mean of its } \\
\text { extreme points) under presumption that } \\
\text { estimate aggregation is conducted using } \\
\text { Borda method }\end{array}$ & 0.1123 & 0.1278 & 0.0813 & 0.1325 & 0.3711 & 0.1351 \\
\hline $\begin{array}{l}\text { Weight ranges under presumption that } \\
\text { estimate aggregation is conducted using } \\
\text { Condorcet method }\end{array}$ & & & & & & \\
\hline $\begin{array}{l}\text { Average weights (weight acceptability area } \\
\text { centre calculated as arithmetic mean of its } \\
\text { extreme points) under presumption that } \\
\text { estimate aggregation is conducted using } \\
\text { Condorcet method }\end{array}$ & & $(0 ; 0.667)$ & $(0 ; 0.238)$ & $(0 ; 0.435)$ & $(0 ; 0.750)$ & $(0 ; 0.400)$ \\
\hline Exact weight values (for comparison) & 0.538 & 0.092 & 0.092 & 0.095 & 0.155 & 0.028 \\
\hline
\end{tabular}


As we can see, the weight values calculated using non-fuzzy rankings differ from the exact ones. Exact weight vector does not even belong to the weight area, obtained based on non-fuzzy rankings (although some of the calculated weight values, such as weight of $C_{3}$ are quite close to exact ones). Such a difference (quite predictable, in fact) results from several factors. First of them is the problem formulation itself. When criterion weights are calculated based on fuzzy rankings (as in section 4), we are searching for specific values, allowing to get as close to the global fuzzy ordinal pair comparison values as possible during mono-criterion estimate aggregation. And in case when only non-fuzzy rankings are given a priori (as in section 2), we can only search for weights' acceptability range, and any value within this range is a problem solution. Secondly, it should not be forgotten, that fuzzy ranking aggregation procedure is different from the rules used for estimate aggregation in Borda and Condorcet methods. And, finally, fuzzy and non-fuzzy rankings bear different kinds of information. Turning to the fuzzy ranking matrix building methodology, suggested by (Jiao \& Tseng, 1998) we can see that it is much more complicated than a series of simple questions, used to obtain normal alternative rankings from the experts. In the example described by Jiao \& Tseng alternative estimation criteria are divided into two sets: tangible and intangible ones. To get alternative estimates according to tangible criteria only measurement data is used. Experts are involved only in the process of alternative ranking according to intangible criteria (i.e. the ones, which can not be interpreted in terms of quantitative measurements). For alternative estimation according to intangible criteria linguistic seven-level scale suggested by (Chen et al., 1992) and simple triangular membership functions, derived from this scale, are used. Obviously, experts' estimates, given in this scale, would bear information of different sort than just ordinal pair comparisons of alternatives.

\section{Further approach extension}

Although fuzzy rankings allow capturing uncertainty in expert judgements, they are more difficult to obtain (than non-fuzzy ones): fuzzy ranking procedure requires more effort from experts and expertise organizers. Many approaches to ranking of fuzzy numbers and to their implementation in decisionmaking support were suggested, studied and compared by different authors (for example, Chen et el. (1992), Jiao \& Tseng (1998), Deep, Kansal \& Singh (2007)). In any case, to build rankings of fuzzy numbers, experts are required to input some numerical (cardinal) values of alternative estimates, which are then used to build membership functions. If experts were inputting ordinal pair comparison values directly into matrices, the procedure would be simplified, and in this case they might as well use the fundamental [1..9] scale for building alternative rankings. Pair comparisons given in fundamental scale can express the degree of expert's confidence in one alternative's dominance over another (and not necessarily reflect the actual cardinal dominance value).

Geometric mean is one of the most commonly used (although, as Choo \& Wedley (2004) show, not always the best in terms of stability) methods of individual and mono-criterion pair comparison matrices aggregation in AHP. The geometric mean method can be transformed into simple weighted summing, if we express expert judgements as logarithms of fundamental scale values. Besides, this would also allow to extend the aforementioned method of weight acceptability area search, inverse to "weighted" Condorcet's aggregation procedure to the case, when "ordinal" pair comparisons lie within the whole range between -1 and $1:\{1 / 9 ; 1 / 8 ; \ldots ; 1 / 2 ; 1 ; 2 ; \ldots ; 9\}$ will be transformed to $\left\{\log _{9} 1 / 9 ; \ldots ; \log _{9} 1 ; \ldots ; \log _{9} 9\right\}$ or $\{-1 ;-0.95 ; \ldots ;-0.32 ; 0 ; 0.32 ; \ldots ; 1\} .9$ is the maximal value from Saaty's scale range, and that is why it is suggested as the logarithm base.

Let us consider a hypothetical numeric example. Say, 4 alternatives $A_{1}-A_{4}$ are estimated according to 3 criteria $C_{1}-C_{3}$ and according to the global criterion $G$. Say, alternative estimates according to criterion $G$ are provided as strict ranking: $\left\{A_{1}, A_{2}, A_{3}, A_{4}\right\}$ and alternative estimates according to criteria $C_{1}-C_{3}$ are provided in the form of ordinal pair comparison matrices, representing both ordinal expert preferences and experts' confidence in these preferences. Say, it is necessary to find a weight vector allowing to preserve global strict ranking of alternatives after mono-criterion pair comparison matrices' aggregation according to "weighted" Condorcet method. Such problem formulation is, in a way, a composition of formulations provided in sections 2 and 4 of this paper. Respective data is provided in table 4. 
Table 4

\begin{tabular}{|l|l|l|l|l|l|l|l|l|l|l|l|l|l|l|}
\hline & \multicolumn{4}{|c|}{ Criterion $C_{1}$} & \multicolumn{4}{c|}{ Criterion $C_{2}$} & \multicolumn{3}{c|}{ Criterion $C_{3}$} \\
& $A_{1}$ & $A_{2}$ & $A_{3}$ & $A_{4}$ & $A_{1}$ & $A_{2}$ & $A_{3}$ & $A_{4}$ & $A_{1}$ & $A_{2}$ & $A_{3}$ & $A_{4}$ & $\begin{array}{l}\text { of alter- } \\
\text { natives according to } \\
\text { criterion } G\end{array}$ \\
\hline$A_{1}$ & 0 & 0.63 & 1 & 0.63 & 0 & -0.89 & -0.73 & -0.95 & 0 & 0.95 & -0.73 & -0.95 & 4 \\
\hline$A_{2}$ & -0.63 & 0 & 0.32 & 0 & 0.89 & 0 & 0 & 0 & -0.95 & 0 & -1 & -1 & 3 \\
\hline$A_{3}$ & -1 & -0.32 & 0 & -0.32 & 0.73 & 0 & 0 & -0.32 & 0.73 & 1 & 0 & -0.32 & 2 \\
\hline$A_{4}$ & -0.63 & 0 & 0.32 & 0 & 0.95 & 0 & 0.32 & 0 & 0.95 & 1 & 0.32 & 0 & 1 \\
\hline
\end{tabular}

Using the method mentioned in section 3 of this paper, we can find criterion weight acceptability area (as a solution of linear inequalities' system built based on mono-criterion pair comparison matrices): its extreme points are $W_{1}=(0.4132,0.4174,0.1694) ; W_{2}=(0.0000,0.3909,0.6091) ; W_{3}=(0.0000$, $0.9030,0.0970)$ and its centre is: $W=(0.1377,0.5704,0.2918)$.

\section{Conclusions}

The key conclusion which can be drawn is that, although none of the individual or single-criterion rankings' aggregation procedures is perfect, normal and fuzzy ordinal estimation experience can and should be used as the data source for weight coefficients' calculation, when it is problematic or impossible to obtain any other estimates beside ordinal ones. Procedure of criterion weight calculation based on non-fuzzy (normal) multi-criteria rankings of alternatives allows to find the acceptability area of weight vectors, preserving alternative ranking according to global criterion after weighted summing of mono-criterion alternative rank scores. The problem of criterion weight calculation based on fuzzy alternative rankings according to several criteria can also be reformulated as a linear programming type problem, although it has only one solution instead of the whole range. Results of criterion weight calculation based on non-fuzzy and fuzzy multi-criteria alternative rankings differ from each other due to the fact that fuzzy rankings bear information of different sort. At the same time, fuzzy alternative ranking procedure is more time-consuming and requires more effort from experts and expertise organizers, even if most calculations are performed by computer software. Weight calculation procedure based on multi-criteria ordinal alternative estimation experience can be modified for cases when aggregate preference relation is built using "weighted" Condorcet and Borda methods. In case experts cannot provide definite answers as to ordinal preference relations between alternatives, they can be offered to input their degree of confidence expressed as logarithm of Saaty's fundamental scale values into pair comparison matrices. Based on these values criterion weights allowing to preserve strict alternative ranking according to a global criterion can be found. This approach is suggested as a simplified alternative to fuzzy ranking based methods. Weight calculation procedures using normal and fuzzy multi-criteria alternative ranking experience as input data can and should be integrated into mathematical software of existing and new decision-making support systems, and thus, expand these systems' functionality.

\section{REFERENCES}

Chen, S. J. et al. (1992). Fuzzy Multiple Attribute Decision Making: Methods and Applications, Berlin; Hong Kong: Springer-Verlag.

Choo, E. U. \& Wedley, W. C. (2004). A common framework for deriving preference values from pairwise comparison matrices. Computers and Operations Research 31, 893-908.

Deep, K., Kansal, M. L. \& Singh, K. P. (2007). Ranking of alternatives in fuzzy environment using integral value, Journal of Mathematics, Statistics and Allied Fields, 1(2):

http://www.scientificjournals.org/journals2007/articles/1234.pdf

Jiao \& Tseng (1998) Fuzzy Ranking for Concept Evaluation in Configuration Design for Mass Customization. Concurrent Engineering: Research and Application. 6(3), 189-206. 


\section{S. Kadenko, Criterion Weights}

Kadenko, S. V. (2009). Experience-based Decision Support Methods Using Ordinal Expert Estimates. Proceedings of the Tenth International Symposium for the AHP/ANP: http://isahp.org

Kadenko, S. V. (2010). Kadenko, S. V. Personnel-related decision-making using ordinal expert estimates. OR Fifty-two Keynotes and Extended Abstracts, Royal Holloway University of London, 177-183.

Saaty, T. L. (2008). The Analytic Hierarchy/Network Process. RACSAM 102 (2), 251-318.

Totsenko, V. G. (2005). Method of Determination of Group Multi-criteria Ordinal Estimates with Account of Expert Competence. Journal of Automation and Information Sciences 37(10), 19-23.

Tseng, T. Y. \& Klein C. M. (1989). New Algorithm for the Ranking Procedure in Fuzzy DecisionMaking. IEEE Transactions on Systems, Man and Cybernetics, 19(5), 1289-96. 